MATEC Web of Conferences 4, 05003 (2013)

DOI: $10.1051 /$ matecconf/20130405003

(C) Owned by the authors, published by EDP Sciences, 2013

\title{
Control of the molecular density in a chemically adsorbed thiophene system monolayer
}

\author{
Yosuke Tanaka, Tadashi Ohtake, and Kazufumi Ogawa \\ Department of Advanced Materials Science, Graduate School of Engineering, Kagawa University, 2217-20 Hayashi-cho, \\ Takamatsu, Kagawa 761-0396, Japan \\ E-mail : s12g564@stmail.eng.kagawa-u.ac.jp
}

\begin{abstract}
Chemically absorbed monolayers (CAM) of thienyl functionalized n-alkyltrichlorosilane 11-(3thienyl) undecyltrichlorosilane (TUTS) have been prepared with two methods which are the standard technique (TUTS-STD) and the twice adsorption method (TUTS-TAM). The existence of TUTS-CAM was confirmed with water contact angle measurements, calculation of thickness with ellipsometry, fourier transform infrared reflection adsorption spectroscopy (FTIR-RAS). Here are described is the comparison of TUTS-STD and TUTS-TAM characterized by using Electron Spectroscopy for Chemical Analysis (ESCA) and ultraviolet visible (UV-vis) absorption spectroscopy. TUTS-TAM showed more densely packing than TUTS-STD by these measurements. Moreover, these TUTS-CAM polymerized by chemical oxidative polymerization and UV-Vis absorption spectra was measured to confirm the conjugated bond length of the polymerized thienyl groups. UV-vis spectra of the polymerized TUTS-CAM showed a new broad absorbance band at longer wavelength than $700 \mathrm{~nm}$
\end{abstract}

\section{Introduction}

Chemically absorbed monolayers (CAM) are closely packed monomolecular films that can be used to modify different substrates surface. Moreover, it was reported in other studies that the molecular density of 14bromotrichlorosilane-CAM was increased by twice adsorption method (TAM) [1]. The first chemical adsorption of TUTS-TAM was conducted with less time than the time to form saturated CAM in order to leave the non-adsorbed portion on the substrates surface. And then, the second chemical adsorption was conducted to fill the non-adsorbed portion. In this paper, we have tried to prepare the high molecular density TUTS-TAM. Finally, the polymerization degrees of TUTS-TAMs and TUTSSTD depending on the molecular density were compared.

\section{Materials and Methods}

\subsection{Materials}

11-(3-thienyl) undecyltrichlorosilane (TUTS) which has a thienyl group at the molecular end, a trichlorosilyl group at the other end, and a hydrocarbon group at the middle portion was synthesized by NARD institute, Ltd [2]. Acetone, chloroform, acetonitrile (Wako Pure Chemical
Industries Ltd), Ethanol (Japan Alcohol Trading co., Ltd) and iron (III) chloride (Merck KGaA) were used as received. Chloroform, acetonitrile and dimethyl silicone fluid (Shin-Etsu Chemical Co., Ltd) were dried by molecular sieves 4A (nacalai tesque) prior to use.

\subsection{Pre-treatment of the substrates}

Prior to the preparation of monolayer, the substrate were sonicated in chloroform, acetone and ethanol, followed by drying in a dry air, and cleaning with excimer lamp (UER20-172B, Ushio INC).

\subsection{Standard technique}

Adsorption of TUTS on the substrates was carried out in dry nitrogen atmosphere. The pre-treated substrates were immersed in the fresh TUTS solution $(0.01 \mathrm{M})$ diluted with dimethyl silicone for 120 minutes to prepared TUTS-STD, and then the substrate was rinsed with copious amounts of chloroform and acetone, and dried in a dry air and held in a laboratory conditions for 24 hours. 


\subsection{Twice adsorption method}

Preparation of the high density TUTS-CAMs on the substrates surface were carried out in dry nitrogen atmosphere. The pre-treated substrates were immersed in the fresh TUTS solution $(0.01 \mathrm{M})$ diluted with dimethyl silicone for 10, 30 and 60 minutes to prepare first loosely packed TUTS-CAMs, and then the substrate were rinsed with copious amounts of chloroform and dried in a dry air and held in a laboratory conditions for 24 hours. Moreover, these substrates covered with the first TUTSCAMs were immersed in the fresh TUTS solution $(0.01$ M) diluted with dimethyl silicone for 120 minutes to increase the molecular density of TUTS-CAMs and then the high density TUTS-CAMs were rinsed with copious amounts of chloroform and acetone, and dried in a dry air and held in a laboratory conditions for 24 hours.

\subsection{Polymerization of TUTS-CAM}

An oxidant solution of iron (III) chloride (0.224 g, $\mathrm{FeCl}_{3}$, $1.38 \mathrm{mmol})$ was prepared with acetonitrile $(50 \mathrm{~mL})$. All TUTS-CAMs on the substrate were polymerized in the oxidant solution in dry nitrogen atmosphere for 10 minutes. The polymerized TUTS-CAMs were rinsed with acetonitrile, and dried under a stream of nitrogen gas.

\subsection{Characterization of TUTS-CAM}

The water droplet contact angles of $3.0 \mu \mathrm{L}$ on the silicon wafers covered with the TUTS-CAMs were measured using the contact angle meter (CA-VP150, Kyowa Interface Science Co., Ltd). The water droplet contact angles were calculated from the CCD camera image of the droplet with integrated multi-functional soft-ware (FAMAS, Kyowa Interface Science Co., Ltd). The water used for contact angle measurements was obtained from the water purification equipment (PURE LITE, organo corporation).

The surface oxide thickness of the silicon wafers used and the thickness of the TUTS-CAMs on the silicon wafers were measured with the DHA-XA/M8 automatic ellipsometer (Mizojili Optical Co., Ltd) attached a He-Ne laser $(\lambda: 632.8 \mathrm{~nm}, 0.8 \mathrm{~mW})$ at incident angles of $70^{\circ}$. In order to obtain an accurate determination of film thickness, the optical constants of $\mathrm{SiO}_{2}(\mathrm{n}: 1.460, \mathrm{k}: 0)$ and TUTS-CAM ( $\mathrm{n}: 1.488, \mathrm{k}: 0)$ were used.

The infrared reflection absorption spectra (IR-RAS) of TUTS-CAMs on the sputtered $\mathrm{SiO}_{2} / \mathrm{Al}$ glass substrate were obtained in the wavenumber region of 3000$2800 \mathrm{~cm}^{-1}$ using FT-IR spectrometer (Nicolet 8700 FT-IR Spectrometer, Thermo Fisher Scientific). The incident light was polarized in S-polarization by the polarizer (ZnSe Polarizer for FT-80, Thermo Electron Co.).

The ESCA spectra of TUTS-CAMs on the silicon wafers were measured on a VersaProbe PHI 5000 Scanning ESCA Microprobe (ULVAC-PHI, inc) attached monochromatic Al-Ka X-ray source. The $\mathrm{C} 1 \mathrm{~s}$ signal due to the carbon chain of TUTS at $284.6 \mathrm{eV}$ was used as the energy reference to correct for the charging.
UV-vis absorption spectra of TUTS-CAMs on the quartz glass ware taken with the UV-vis spectrophotometer (UV-1800, Shimadzu Corporation) .

\section{Results and Discussion}

\subsection{TUTS-CAM by the standard technique}

In order to optimize the preparation conditions for manufacturing the homogeneous and smooth TUTSCAM, the immersion times were changed at different time. Figure 1 shows that the water contact angles and thickness change of TUTS-CAMs measured. The contact angles and the thickness increased with increasing the immersion times, and levelled off over 120 minutes.

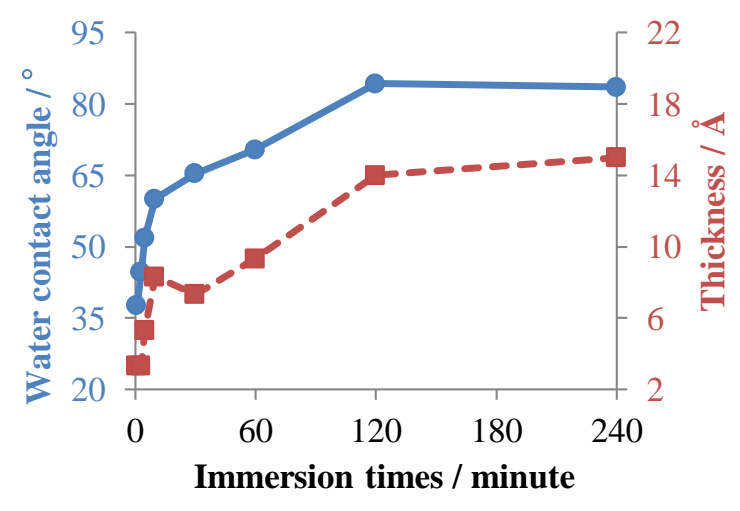

Fig. 1. Water contact angle (Solid line) and thickness (Dashed line) changes of TUTS-CAMs as a function of the immersion times.

Figure 2 shows IR-RAS of TUTS-CAMs. The asymmetric stretching vibration modes due to $\mathrm{CH}_{2}$ $\left(v_{\mathrm{as}} \mathrm{CH}_{2}\right)$ were appeared at around $2920-2930 \mathrm{~cm}^{-1}$; and the symmetric stretching vibration modes due to $\mathrm{CH}_{2}$ $\left(v_{\mathrm{s}} \mathrm{CH}_{2}\right)$ were appeared at around 2850-2860 $\mathrm{cm}^{-1}$ [3]. The wave number of the $v_{\mathrm{as}} \mathrm{CH}_{2}$ shifted to the red direction with increasing the immersion times, and the red shift levelled off over 120 minutes, as shown in Fig. 3. This indicate that the molecular density of TUTSCAM were densely and fully packed over 120 minutes. The infrared absorption frequency of the monolayer is generally calculated using the equation (1) [1]

$f=\frac{1}{2 \pi c} \sqrt{\frac{K}{\mu}}$

where in, $\mathrm{f}$ is the infrared absorption frequency, $\pi$ is the circle constant, $c$ is the velocity of light, $K$ is the constant of bond strength and $\mu$ is the reduced mass. The packing density of the carbon chain becomes higher, the bond stretching of $v_{\mathrm{as}} \mathrm{CH}_{2}$ becomes less. That is, the wavenumber of $v_{\mathrm{as}} \mathrm{CH}_{2}$ peak on the infrared spectra decreased, the molecular density of TUTS-CAM increased. Thus, Fig. 3 shows that the TUTS molecules in the TUTS-CAMs prepared over 120 minutes are densely and fully packed. 


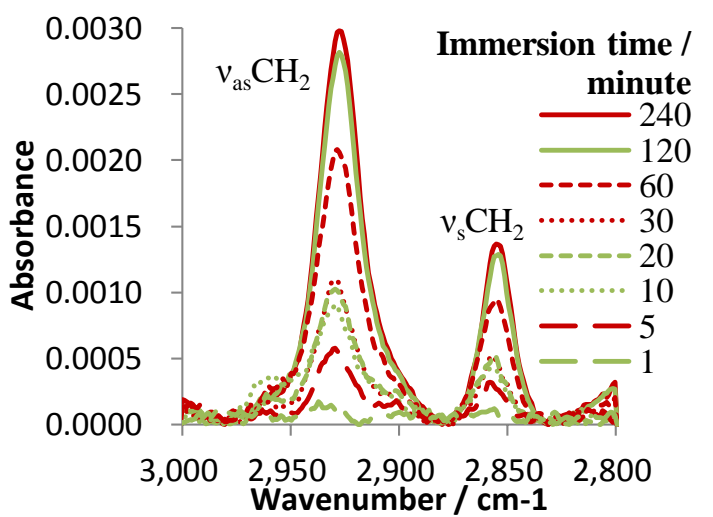

Fig. 2. IR-RAS changes of TUTS-CAMs with increasing the immersion times.

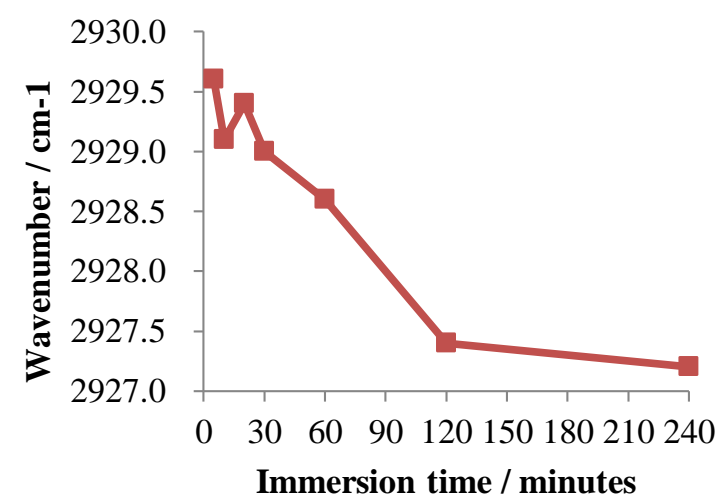

Fig. 3. The wave number shifts of the absorption bands due to $v_{\text {as }} \mathrm{CH}_{2}$ show in Fig. 2.

\subsection{TUTS-CAMs by twice adsorption method (TAM)}

According to the TUTS-CAM preparation condition for the standard technique (TUTS-STD; the immersion time was $120 \mathrm{~min}$ ), firstly, the pre-treated substrates were less immersed in the fresh TUTS solution $(0.01 \mathrm{M})$ diluted with dimethyl silicone for 10,30 and 60 minutes to prepare the loosely packed monolayer of TUTS-CAM. Secondly, these samples were immersed in the fresh TUTS solution $(0.01 \mathrm{M})$ diluted with dimethyl silicone for 120 minutes to prepare the densely and fully packed TUTS-CAM.

The water contact angles and thickness of TAM60 were $82.1^{\circ}$ and $16.0 \AA$, respectively. Therefore, the water contact angles and thickness of the TUTS-CAM with TAM60 was changed a little, when compared with the TUTS-STD.

Figure 4 shows the UV-vis absorption spectra of TUTS-CAMs. The UV-vis absorption spectroscopy is useful to quantitative measure the thienyl groups in TUTS-CAM. The UV-vis absorption spectra of all TUTS-CAMs showed the sharp absorption bands at the wavelengths of $235 \mathrm{~nm}$. These absorption bands originated from the conjugated bond of the thienyl group. Figure 5 shows the absorption band area ratios of TUTSTAMs to TUTS-STD between $210 \mathrm{~nm}$ and $260 \mathrm{~nm}$ due to TUTS-TAMs and TUTS-STD. TAM60 was the largest



Fig. 4. UV-vis absorption spectra of TUTS-TAMs prepared at 10, 30, 60minutes and TUTS-STD before the polymerization.



Fig. 5. The UV-vis absorption band area ratio changes of TAM10, TAM30 and TAM60 to that of STD between $210 \mathrm{~nm}$ and $260 \mathrm{~nm}$ in Fig. 4.

band area of all samples; therefore, TAM60 possessing the most thienyl groups on all TUTS-CAMs.

Figure 6 shows the ESCA spectra of four TUTSCAMs. $S 2 p_{3 / 2}$ and $S 2 p_{1 / 2}$ components of the $S 2 p$ core level of thienyl group appeared at 164.2 and $165.4 \mathrm{eV}$, respectively [4]. Moreover, the band areas of $S 2 p_{3 / 2}$ of three TAMs were compared to that of STD. The band area of TAM60 was the largest on all samples similar to the UV-vis spectra. Therefore Fig. 7 shows that TAM60 possesses the most thienyl groups on all TUTS-CAMs. That is, TAM60 is the highest molecular density TUTSCAM of all.

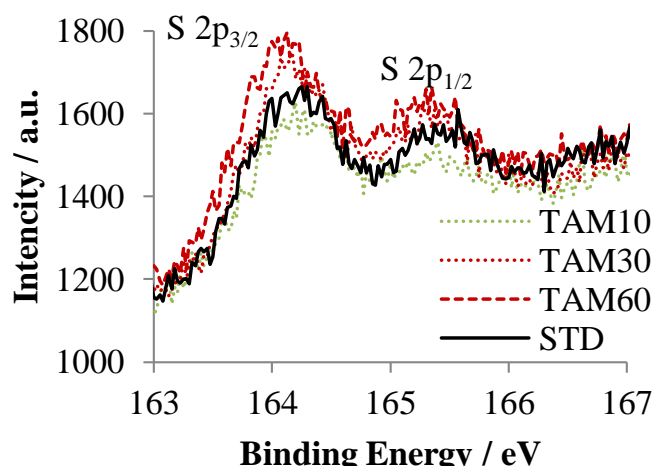

Fig. 6. ESCA spectra of TAM10, TAM30, TAM60 and STD of TUTS-CAM. 


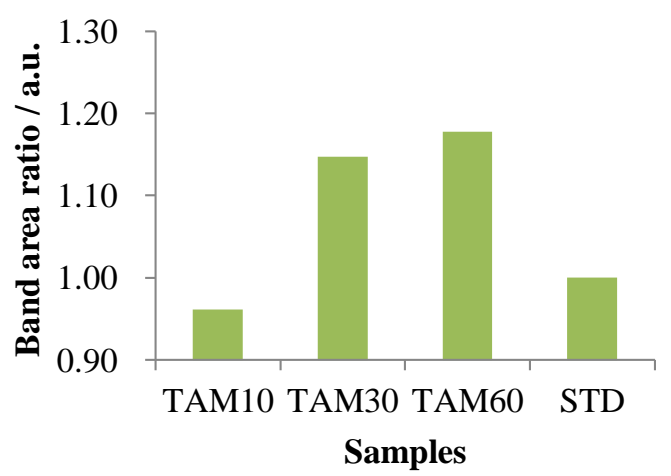

Fig.7. The band area ratio changes of $S 2 p_{3 / 2}$ of TAM10, TAM30 and TAM60 to that of STD in Fig. 6.

\subsection{Polymerization of TUTS-CAM}

Figure 8 shows the UV-vis absorption spectra of the polymerized four TUTS-CAMs. New broad absorption bands appeared at $350 \mathrm{~nm}, 700 \mathrm{~nm}$ and over $1100 \mathrm{~nm}$ in all spectra. These absorption bands appeared on the polymerized TUTS-CAM indicate that the super long conjugated bonds of the poly-thienyl groups may be prepared by this polymerization technique of TUTSCAMs [5, 6]. Moreover, the band area between 520nm to $900 \mathrm{~nm}$ of three TAMs were compared with that of STD, as shown in Fig. 9. The band area of TAM60 was the largest on all samples, indicating that the polymerized thienyl groups of TAM60 possesses the most long conjugated bond. The long conjugated bond was increased a little with increasing the TUTS molecular density. However the peak absorption wavelength was not changed on all samples $(741.0 \mathrm{~nm})$, indicating that the conjugated bond length was not changed on all samples.

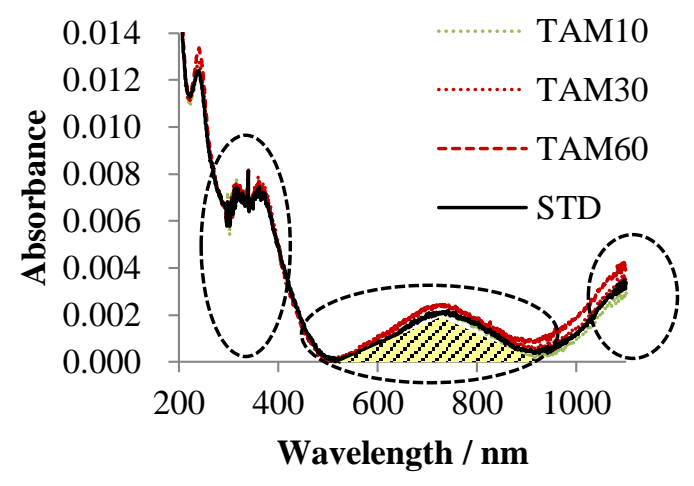

Fig. 8. UV-vis absorption spectra of polymerized TAM10, TAM30, TAM60 and STD of TUTS-CAM after the polymerization.

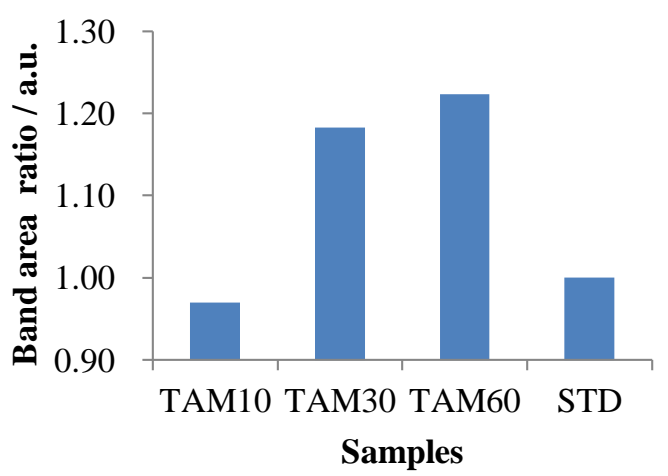

Fig. 9. The absorbance band area ratio changes of TAM10, TAM30 and TAM60 to that of STD between $520 \mathrm{~nm}$ and $900 \mathrm{~nm}$ in Fig. 8.

\section{Conclusion}

The most suitable adsorption time to prepare the densely packed TUTS-CAM was 120 minutes by standard technique (STD). The molecular density of TUTS-CAM was more increased by the twice adsorption method (TAM) than by STD. The conjugated length of polymerized thienyl groups of TUTS-TAM increased slightly with increasing the TUTS molecular density.

\section{Acknowledgements}

The authors would like to thank Prof. Fumikazu Oohira for his a assistance on the ellipsometry and Prof. Naoshi Takahashi for his a assistance on the ESCA.

\section{References}

1. Tadashi Ohtake, Studies on Structure and Properties of Chemically-Adsorbed Layers, Kagawa University (2010)

2. D. Appelhans, D. Ferse, H.-J.P. Adler, W. Plieth, A. Fikus, K. Grundke, F.-J. Schmitt, T,Bayer, B.Adolphi, Colloids Surf., A, 161, 203-212 (2000)

3. Arthur D.Quest, Nathan C. Wilde, Sam S.Matthews, Scott T.Maughan, Steven L. Castle, James E.Patterson, Vibrational Spectroscopy 61, 17-24 (2012)

4. Agata Blacha, Piort Koscielniak, Michal Sitarz, Jacek Szuber, Jerzy Zak, Electrochemica Acta 62, 441-446 (2012)

5. P.Sivaraman, Sarada P. Mishra, Arup R.Bhattacharrya, Avinash Thakur, K. Shashidhara, Asit B.Samui, Electrochemica Acta 69, 134-138 (2012)

6. Tsuyoshi Izumi, Seiji Kobashi, Kazuo Takimiya, Yoshio Aso, Tetsuo Otsubo, JACS (2003) 